\title{
Tuning of laser pulse shapes in grating-based compressors for optimal electron acceleration in plasmas
}

\author{
Cs. Tóth, J. Faure, J. van Tilborg, C. G. R. Geddes, C. B. Schroeder, E. Esarey, and W. P. Leemans \\ Lasers Optics and Accelerator Systems Integrated Studies (L'OASIS) Group, Accelerator and Fusion Research Division, \\ Lawrence Berkeley National Laboratory, Building 71R0259, 1 Cyclotron Road, Berkeley, California 94720 \\ Received January 22, 2003 \\ The temporal shape (rise time, fall time, skewness) of 50-200-fs Ti:sapphire laser pulses has been controlled \\ by appropriate adjustment of a grating-pair compressor. It was found that the skewness of the laser pulse \\ envelope is particularly sensitive to the third-order component of the spectral phase. Introducing such a \\ third-order phase offset by detuning the grating pair relative to the optimum pulse compression settings \\ allowed the generation of skewed pulses. As an example of an application, these skewed pulses were used \\ to optimize a laser-plasma electron accelerator. (c) 2003 Optical Society of America \\ OCIS codes: $320.5520,320.5540,320.7160,350.5400$.
}

The details of the time-envelope function of ultrashort light pulses (pulse shape) can play a crucial role in many nonlinear optical phenomena. Two light pulses with the same full width at half-maximum (FWHM) but different rise- and fall-time characteristics, or amplitude modulation, can lead to vastly different results in processes in which the excitation in the medium depends strongly on the instantaneous light intensity. An example is the yield and energy spectrum of accelerated electrons in a self-modulated laser wake-field accelerator (SM-LWFA), ${ }^{1}$ where a driving pulse with a sharp leading edge will result in faster growth of the plasma wake field, and consequently, higherenergy electrons after acceleration. The extreme sensitivity of these types of nonlinear process to pulse shape calls for deeper understanding of the pulse-forming mechanisms in laser amplifier systems and for new, simple methods of pulse-shape manipulation. There are several methods of actively controlling the shape of ultrashort light pulses, such as frequencydomain filtering and spectral-time-domain envelope transfer by use of liquid-crystal phase modulators ${ }^{2}$ and acousto-optic phase and amplitude control. ${ }^{3}$ In this Letter we investigate the intrinsic pulse-shaping behavior of the most widely used optical pulse compressor-the grating pair-with emphasis on pulse skewness and its effect on the strongly nonlinear process of SM-LWFA.

The conventional description of the propagation and phase evolution of ultrashort light pulses and the related dispersive delay in each optical component of a laser system uses a Taylor series expansion of the optical phase around the center angular frequency, $\omega_{0}$. In this expansion the coefficients represent the linear chirp (group-delay dispersion, second order), the cubic phase (third order), the quartic phase, and so on. The properties of ultrashort $(<100-\mathrm{fs})$ optical pulses strongly depend on these higher-order phase terms. In contrast to the simplest case of pure linear chirp, the presence of higher-order components modifies the shape of the pulse, resulting in modulation and even in the appearance of prepulses or postpulses. ${ }^{4-7}$ To characterize quantitatively the shape of the pulse, we introduce the skewness parameter, $S$. A simple definition of $S$ could be based on the ratio of the tail width at half-maximum (TWHM) and the head width at half-maximum (HWHM) of the laser intensity, as $S=($ TWHM/HWHM) -1 . In the case of laser-plasma accelerators, another definition of skew based on second- and third-order moments proved to be useful:

$$
\begin{aligned}
S & =m_{3} / m_{2}{ }^{3 / 2}, \\
m_{k} & =\int_{a}^{t_{b}}\left(t-t_{0}\right)^{k} I(t) \mathrm{d} t / \int_{a}^{t_{b}} I(t) \mathrm{d} t,
\end{aligned}
$$

where $I(t)$ is the laser intensity temporal profile and $t_{0}$ is the centroid. The contours in Fig. 1 summarize the main types of skewed pulses: $S=0$ corresponds to a symmetric pulse shape, $S<0$ describes a pulse with a slowly increasing front part and a suddenly dropping tail, and $S>0$ represents a fast increase and a long tail.

In a typical chirped-pulse amplification (CPA) system, the final pulse-forming device is the grating-pair pulse compressor. Scanning the grating separation not only changes the pulse length and the linear chirp but also modifies all the higher-order terms. ${ }^{6,7}$ The effects of these slight changes on the pulse shape become negligible at settings far from the shortest compression, i.e., where the pulse duration is much longer than the shortest possible. However, when the pulse duration is in the range of a few times the minimum and the compressor angle is set slightly differently from the optimum, or when the pulse contains higher-order terms,
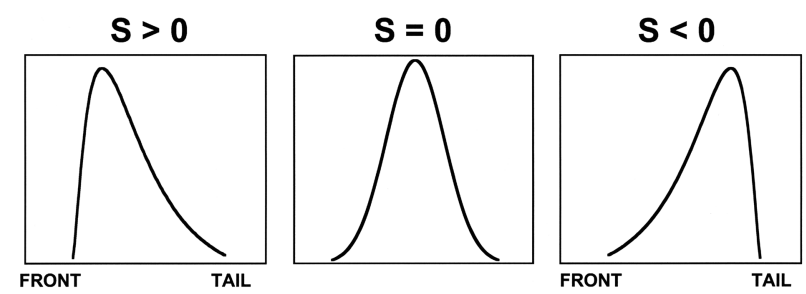

Fig. 1. Typical skewed pulse shapes characterized by the skewness parameter, $S$. 
a typical scan around the shortest pulse will produce significant changes in the pulse shape.

For example, compressing stretched pulses with a positive-bias third-order phase component will initially skew the pulse shape in the course of a compressor separation scan toward the head of the pulse $(S>0)$, and then flip it to become skewed at the tail $(S<0)$, and finally flip it back to the original asymmetric shape $(S>0)$ again, as plotted in Fig. 2(a). We note that these changes of the pulse envelope are fundamentally different from the well-known mapping of the spectral amplitude to the time domain for strongly chirped pulses, where only the dominant, second-order phase coefficient plays any role. ${ }^{2}$ Further details of this skew-flip behavior can be seen in Fig. 2, which shows the simulated pulse shape dependence for a light pulse of $\sim 20$-nm spectral bandwidth during a grating compressor scan around the shortest pulse. The grating parameters are groove density of $1480 \mathrm{~mm}^{-1}$, angle of incidence of $50.0^{\circ}$, grating separation at the shortest pulse $323 \mathrm{~mm}, \lambda=795 \mathrm{~nm}$, and third-order phase bias $\phi_{3}=+30,000 \mathrm{fs}^{3}$ [Fig. 2(a)], $\phi_{3}=0 \mathrm{fs}^{3}$ [Fig. 2(b), and $\phi_{3}=-30,000 \mathrm{fs}^{3}$ [Fig. 2(c)]. A third-order phase change of $30,000 \mathrm{fs}^{3}$ can be achieved by tuning both gratings of the compressor by $\sim 0.6^{\circ}$.

In a real laser system, however, the situation is complicated by the nonnegligible presence of even higher-order phase terms beyond the third. The solid curve in Fig. 3 shows a full simulation of the pulse-shape evolution in the Ti:sapphire CPA laser system of the Laser Optics and Accelerator Systems Integrated Studies (L'OASIS) Laboratory at Lawrence Berkeley National Laboratory. The dispersion effects of the Ti:sapphire crystals, all optical materials, and $90-\mathrm{m}$ air were included in the simulations. The squares correspond to measured data, averaged over five laser shots. The pulses were fully characterized by retrieval of the amplitude and phase functions from polarization-gate frequency-resolved optical gating images. ${ }^{8}$ The two insets in Fig. 3 show two example retrievals, one at the positive chirp side (a) and one at the negative chirp side (b). The difference of the pulse shapes on the two sides of the scan is apparent: While both pulses have the same FWHM pulse duration ( $76 \mathrm{fs}$ ), the pulse at the positive chirp side has a sharper rising edge than the pulse with negative chirp, in good agreement with the simulation results.

To illustrate the practical importance of the skewness of ultrashort laser pulses, we describe below the effects of these various pulse shapes in a strongly nonlinear experiment: the acceleration of electrons by laser-produced plasma wakes in He plasma. In SM-LWFA experiments we observed strong asymmetry in the yield of electrons and neutrons produced by the accelerated electrons with energy of multiple lens of mega electron volts as a function of the compressor scans. ${ }^{9,10}$ At optimum compression, a peak power of $8.3 \mathrm{TW}$, a calculated peak intensity of $1.5 \times 10^{19} \mathrm{~W} / \mathrm{cm}^{2}$, and a normalized laser strength of $a_{0}=8.6 \times 10^{-10} \lambda[\mu \mathrm{m}] I^{1 / 2}\left[\mathrm{~W} / \mathrm{cm}^{2}\right]=2.6$ were achieved. The total charge of the emitted electrons [typically 2-5 nC; solid curve in Fig. 4(a)] was found to depend strongly on the position of the grating separation relative to the optimum pulse-compression setting [dotted-dashed vertical line in Fig. 4(a)]. Analytic modeling and simulation indicate that the effect of the intrinsic frequency chirp is less important and by itself cannot explain the observed anomaly. ${ }^{11}$ On the other hand, taking into account the shape dependence, it is possible to explain the observed asymmetric enhancement seen in Fig. 4(a). Plasma waves generated by Raman scattering of the laser pulse in the plasma are responsible for the energetic electron production. ${ }^{12}$ The Raman scattering instability is initially seeded by plasma waves excited by the leading edge of the laser pulse. That is, faster rise times generate larger plasma wakes underneath the laser pulse envelope, resulting in larger Raman plasma waves and a larger electron yield. We note here that the following analysis refers strictly to the SM-LWFA regime corresponding to our experimental parameters ${ }^{13,14}$ and differs from other work related to pulse-shape dependences in the standard wake-field acceleration regime. ${ }^{15}$

To evaluate the Raman instability seed excited by the laser envelope, one may calculate the initial plasma response to the laser pulse for the experimental temporal laser profiles, using the one-dimensional nonlinear quasi-static fluid equations. ${ }^{12}$ In particular, the plasma electrostatic potential $\psi$ evolves via

$$
\frac{\partial^{2} \psi}{\partial \zeta^{2}}=\frac{\omega_{p}^{2}}{2 c^{2}}\left[\frac{\gamma_{\perp 0}^{2}}{(1+\psi)^{2}}-1\right]
$$

where $\zeta=z-c t, \omega_{p}{ }^{2}=4 \pi e^{2} n / m_{e}, n$ is the electron plasma number density, and $\left(\gamma_{\perp 0}{ }^{2}\right)=1+a_{0}^{2} / 2$ is the Lorentz factor that is due to the quiver motion of the electrons in the presence of the laser field. In the near-adiabatic limit the solution to this equation scales as $\delta \psi \propto a_{0}^{2} /\left[\omega_{p} \tau_{R}\left(\gamma_{\perp 0}\right)^{1 / 2}\right]$, where $\delta \psi=\psi+1-\gamma_{\perp 0}$ and $\tau_{R}$ is the rise time of the front of the laser pulse, i.e., $c \partial_{\zeta} a_{0} \sim a_{0} / \tau_{R}$. The source driving the Raman
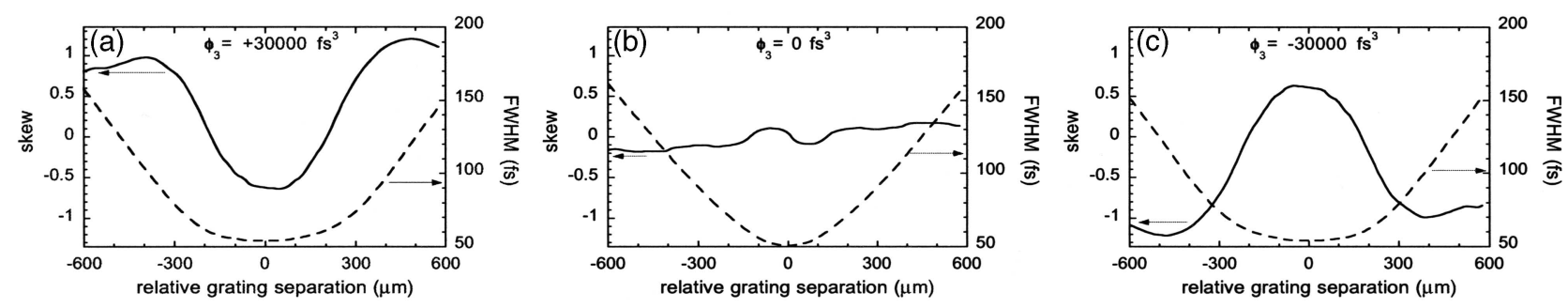

Fig. 2. FWHM (dashed curve) and skew $S$ (solid curve) of ultrashort pulses in a CPA system versus grating separation, simulated for three different third-order bias phase components: (a) $\phi_{3}=+30,000 \mathrm{fs}^{3}$, (b) $\phi_{3}=0 \mathrm{fs}^{3}$, (c) $\phi_{3}=-30,000 \mathrm{fs}^{3}$. 


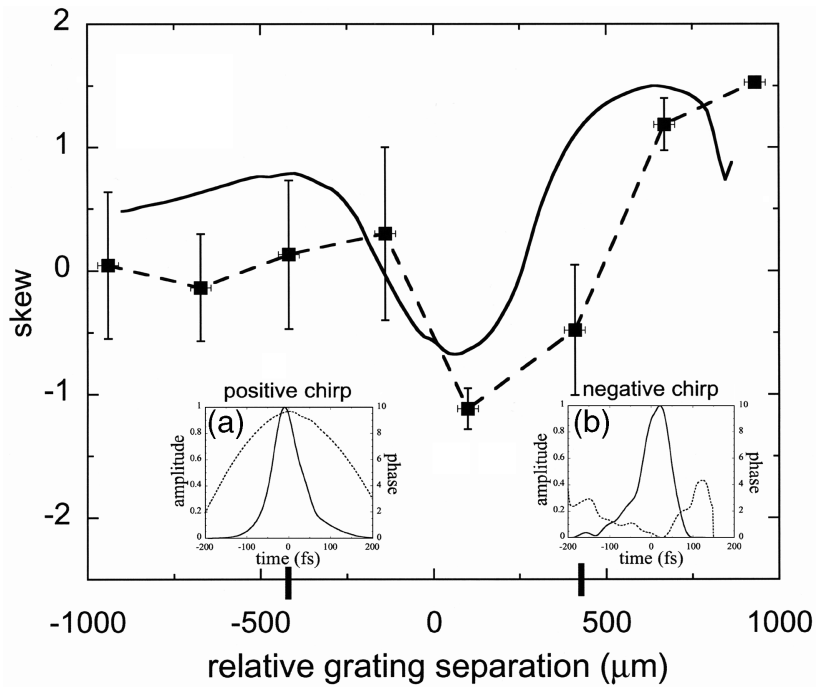

Fig. 3. Simulated (solid curve) and measured (squares) skew of pulses in a CPA system plotted versus grating separation. The insets show retrieved amplitude and phase functions in the time domain at the marked relative grating separations: (a) $-420 \mu \mathrm{m}$ and (b) $+410 \mu \mathrm{m}$.
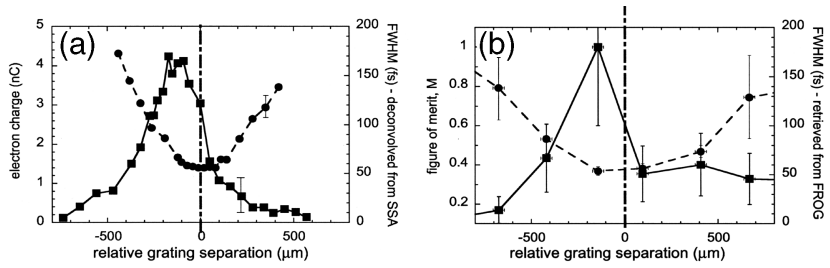

Fig. 4. (a) Measured yield of accelerated electrons (solid curve) and FWHM of the exciting laser pulses (dashed curve) deconvolved from single-shot autocorrelation measurement. (b) Figure of merit $M$ (solid curve and squares), defined by combination of pulse skewness and FWHM, versus compressor position in SM-LWFA experiments. Pulse widths retrieved and averaged from frequency-resolved optical gating measurements (dashed curve and circles) are also plotted in (b). In (a) and (b) the vertical dotted-dashed line represents the shortest pulse-compression position of the gratings.

instability scales as $a_{0} \delta \psi /\left(\gamma_{\perp 0}\right)^{2}$; therefore, one can define a figure of merit $M$ for the seeding of the Raman scattering that scales as $M \propto a_{0}{ }^{3} \tau_{R}{ }^{-1}\left(1+a_{0}{ }^{2} / 2\right)^{-5 / 4}$. Assuming constant laser pulse energy, the laser strength can be expressed as $a_{0}=\left(\tau_{0} / \tau\right)^{1 / 2}$, where $\tau$ is the pulse duration and $\tau_{0}[\mathrm{ps}]=46.5 E[J]\left(\lambda / r_{s}\right)^{2}$, where $r_{s}$ is the laser spot size, $\lambda$ is the wavelength, and $E$ is the pulse energy. Since the rise time of the laser pulse can be expressed in terms of the skew parameter $\tau_{R} \sim \tau /(S+2)$, the figure of merit for seeding of Raman scattering can be written as

$$
M=M_{0}\left(\tau_{0} / \tau\right)^{5 / 2}(S+2) /\left(1+\tau_{0} / 2 \tau\right)^{5 / 4},
$$

where $M_{0}$ is an arbitrary normalization constant.

The calculated figure of merit values (squares) are plotted in Fig. 4(b). The asymmetric nature of this function around the best compression position clearly shows the importance of the skew, and it is well cor- related with the experimentally observed asymmetric electron-yield enhancement.

In conclusion, we have shown that appropriate knowledge and control of the pulse shapes in grating-based CPA systems can be used for optimization of a laser wake-field accelerator. The interplay of pulse shapes and the sign of chirp can lead to enhanced growth of the plasma wake that is responsible for electron acceleration. For our experiments, this enhanced growth results in an optimal acceleration condition that differs from the use of the shortest, highest-intensity pulse. In other words, pulse shapes with sharp leading edges are favored. We achieved pulse shaping with a conventional grating compressor scan by intentionally offsetting the higher-order phase components. This method represents another simple way of higher-order phase control as a convenient alternative to the recently developed acousto-optic phase modulation devices such as the DAZZLER. ${ }^{3,16}$

The authors acknowledge discussions with B. Shadwick, and D. Fittinghoff. This work was partially supported by U.S. Department of Energy grant DE-AC-03-76SF0098. C. Geddes also acknowledges support from the Hertz Foundation. Cs. Tóth's e-mail address is ctoth@lbl.gov.

\section{References}

1. W. P. Leemans, C. W. Siders, E. Esarey, N. E. Andreev, G. Shvets, and W. B. Mori, IEEE Trans. Plasma Sci. 24, 331 (1996).

2. A. M. Weiner, D. E. Leaird, J. S. Patel, and J. R. Wullert, IEEE J. Quantum Electron. 28, 908 (1992).

3. F. Verluise, V. Laude, Z. Cheng, C. Spielmann, and P. Tournois, Opt. Lett. 25, 575 (2000).

4. D. N. Fittinghoff, B. C. Walker, J. A. Squier, C. Tóth, C. Rose-Petruck, and C. P. J. Barty, IEEE J. Sel. Top. Quantum Electron. 4, 430 (1998).

5. S. Backus, C. G. Durfee III, M. M. Murnane, and H. C. Kapteyn, Rev. Sci. Instrum. 69, 1207 (1998).

6. A. Sullivan and W. E. White, Opt. Lett. 20, 192 (1995).

7. J. Squier, C. P. J. Barty, F. Salin, C. Le Blanc, and S. Kane, Appl. Opt. 37, 1638 (1998).

8. R. Trebino, Frequency-Resolved Optical Gating: The Measurement of Ultrashort Laser Pulses (Kluwer Academic, Dordrecht, The Netherlands, 2002), p. 101.

9. W. P. Leemans, D. Rodgers, P. E. Catravas, C. G. R. Geddes, G. Fubiani, E. Esarey, B. A. Shadwick, R. Donahue, and A. Smith, Phys. Plasmas 8, 2510 (2001).

10. W. P. Leemans, P. Catravas, E. Esarey, C. G. R. Geddes, C. Tóth, R. Trines, C. B. Schroeder, B. A. Shadwick, J. van Tilborg, and J. Faure, Phys. Rev. Lett. 89, 174802 (2002).

11. C. B. Schroeder, E. Esarey, B. A. Shadwick, and W. P. Leemans, Phys. Plasmas 10, 285 (2003).

12. E. Esarey, P. Sprangle, J. Krall, and A. Ting, IEEE Trans. Plasma Sci. 24, 252 (1996).

13. V. V. Goloviznin, P. W. van Amersfoort, N. E. Andreev, and V. I. Kirshanov, Phys. Rev. E 52, 5327 (1995).

14. J. Krall, E. Esarey, P. Sprangle, and G. Joyce, Phys. Plasmas 1, 1738 (1994).

15. A. Spitkovsky and P. Chen, Phys. Lett. A 296, 125 (2002).

16. K. Ohno, T. Tanabe, and F. Kannari, J. Opt. Soc. Am. B 19, 2781 (2002). 